\title{
NeuroImage
}

\section{Brain mechanisms for mood congruent memory facilitation}

\author{
P.A. Lewis, ${ }^{*}$ H.D. Critchley, A.P. Smith, and R.J. Dolan \\ Functional Imaging Laboratory, 12 Queen Square, WC1N 3AR, London
}

Received 13 September 2004; revised 4 November 2004; accepted 29 November 2004

Available online 2 March 2005

Emotional information is better remembered when mood at the time of retrieval matches it in valence (positive mood, positive material). An associative memory model predicts that this 'mood congruent' facilitation is due to the mood-related reactivation at retrieval of emotional responses which were linked to valenced information at encoding. To test this model, we presented subjects with positive and negative words at study and manipulated their mood at test while using functional imaging to monitor brain activity. Subjective mood ratings and heart rate variability both indicated that the manipulation was effective, and memory performance showed a strong trend towards facilitation in congruent conditions. In the functional imaging data, valence-specific conjunctions between encoding activity predicting subsequent memory in a congruent mood and retrieval activity relating to mood congruent recollection revealed shared responses in subgenual cingulate for positive valence and posteriolateral orbitofrontal cortex for negative valence, thus supporting the associative model. To elucidate the mnemonic basis of facilitation, independent of valence, we examined the shared correlates of positive and negative congruence and found that parts of the episodic memory system were activated by congruence in correct rejection trials, but no part of this system was activated by congruence in correctly remembered trials. This pattern suggests that mood congruent facilitation occurs at the level of attempted recall rather than that of successful recollection.

(C) 2005 Elsevier Inc. All rights reserved.

Keywords: Mood congruent facilitation; Functional imaging; Valence; Emotion; Memory

\section{Introduction}

Emotional material is preferentially processed in mood states with a matching affective valence (Blaney, 1986; Elliott et al., 2002; Gotlib and Cane, 1987; Murphy et al., 1999; Segal et al., 1995). For example, a happy mood makes it easier to recall memories of positive occurrences or information with positive connotations, while negative memories predominate in sad moods. This mood congruent facilitation is not confined to

* Corresponding author. Fax: +64 44741731.

E-mail address: p.lewis@fil.ion.ucl.ac.uk (P.A. Lewis).

Available online on ScienceDirect (www.sciencedirect.com). episodic memory; similar effects have been documented in other cognitive contexts including Stroop conflict tasks and emotional go/no-go tasks (Elliott et al., 2002; Murphy et al., 1999; see Blaney, 1986 for a review). Elliott et al. (2002) examined the brain activity associated with mood congruent processing in a go/ no-go task, while other studies have examined the brain activity associated with the influence of mood on verbal fluency (Baker et al., 1997) and working memory (Gray et al., 2002). The brain basis for mood-related facilitation in episodic memory, however, remains unclear. Our objective in the current study was therefore to address the neural processes mediating mood congruent effects in memory by using functional magnetic resonance imaging (fMRI) to index the brain activity engaged during both encoding and retrieval phases of a mood congruent memory task.

One dominant theoretical model of emotional context and memory (Bower, 1981) (Fig. 1) predicts that mood-related memory facilitation can be explained as an associative memory effect. According to this model, memories are stored as distributed traces, with different nodes in the trace network representing different aspects of the memory (i.e., the place where it happened, the sounds or smells present, and the emotional valence of the memory). Emotional nodes can be excited by a wide range of stimuli with the appropriate valence, and also by appropriately valenced moods. Once active, these nodes can propagate excitation to the rest of the associated network, bringing the whole trace nearer to the threshold of excitation required for retrieval, and thus facilitating recall. Building upon this framework, we conjectured (Lewis and Critchley, 2003) that it may be possible to index activity corresponding to these nodes using fMRI. Specifically, we proposed that mood congruent memory facilitation is associated with activity in emotion-related brain structures which are both engaged when material of a particular valence is encoded, and reactivated by congruent moods at retrieval. This possibility is supported by functional imaging studies which have provided evidence for an associative link between stored material and the valence of its context at encoding (Maratos et al., 2001). Because facilitation will only occur if material is remembered in a congruent mood, our model predicts greater activity in emotional nodes during congruent than incongruent recall. Furthermore, because facilitation will only occur if emotional nodes were also active at encoding, activity in these areas during encoding should predict subsequent memory in congruent as compared to incon- 


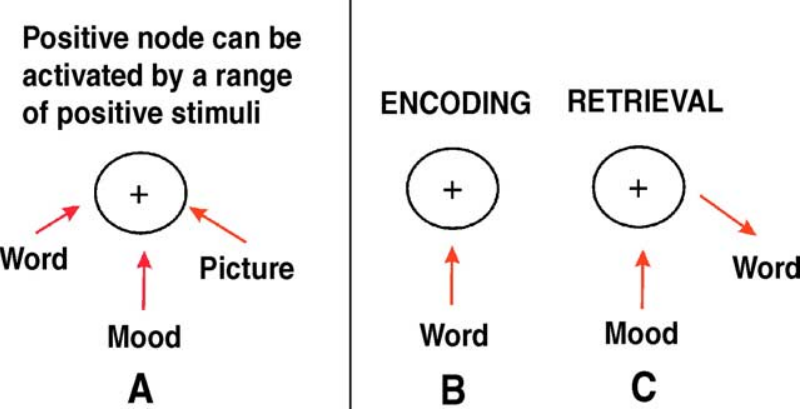

Fig. 1. Associative model for mood congruent memory facilitation. This model proposes the existence of emotion specific 'nodes' (A) which are activated by a range of stimuli with a particular valence. In memory, the node becomes associatively linked to valenced information at encoding (B). At retrieval, the mood state with the same valence as the information to be recalled can activate the node $(\mathrm{C})$ thus lowering the threshold for activation of the memory trace and facilitating recall.

gruent moods. Our analysis draws upon these predictions to isolate candidate emotion-specific nodes.

In addition to valence-specific affects, mood congruent memory facilitation may involve valence-independent alterations in memory processes. These changes could occur either at the level of correct retrieval (Smith et al., 2004), in which case it should be possible to isolate them by examining correctly recollected (RR) items, or at the level of attempted retrieval, in which case it should be possible to isolate them by examining correctly rejected (CR) trials. In these trials, subjects correctly indicate that an item was not present at the time of encoding; thus, a retrieval attempt is made, but does not result in actual memory. Because the different types of processing which can be applied to a retrieval cue are known as retrieval orientations (Hornberger et al., 2004; Robb and Rugg, 2002; Rugg et al., 2003), activity associated with CR responses can be thought of as an index of retrieval orientation (Hornberger et al., 2004; Robb and Rugg, 2002; Rugg et al., 2003). In the current study, we examined the influence of mood congruence on both RR and $\mathrm{CR}$ trials in order to determine its relative impact upon recollection proper and retrieval orientation.

\section{Materials and methods}

Subjects

Eighteen healthy right-handed (English first language) volunteers gave full informed consent to participate in this experiment, which was approved by the Joint Ethics Committee of the National Hospital and Institute of Neurology. Consistent with prior work (Smith et al., 2004), four subjects were excluded due to inadequate performance (false alarm $\mathrm{R}$ response rates greater than $30 \%$ indicating a high level of guessing). Of the remaining 14 subjects, 7 were male and 7 female (mean age was 29 years).

\section{Task design}

We used a task in which subjects encoded positive and negative words and were then tested for episodic memory after each of a series of mood inductions designed to elicit positive and negative moods in a counterbalanced order (see Fig. 2).

At study, a series of words were presented for $1 \mathrm{~s}$ each, with an SOA of $3 \mathrm{~s}$. Stimuli were selected from the ANEW list (Bradley and Lang, 1999), a database of English words with standardized ratings of valence and arousal. 124 words with a valence rating $>7$ (out of 9), for example, 'cute', 'happy', and 124 words with a valence rating $<3$, for example, 'brutal', 'maggot', were shown in a fully randomized order. Subjects performed a self-referential deep encoding task, indicating whether the presented words could be used to describe themselves in any abstract sense. Subjects responded with button presses using the right hand and were not informed that their memory would subsequently be tested.

At test, we used a 'remember/know/new' $(\mathrm{R} / \mathrm{K} / \mathrm{N})$ memory task (Tulving, 1985). Subjects saw a total of 360 words each presented

\section{Sequence of events in task}

\section{Part 1: Training}

\section{words presented every $\mathbf{3}$ seconds}

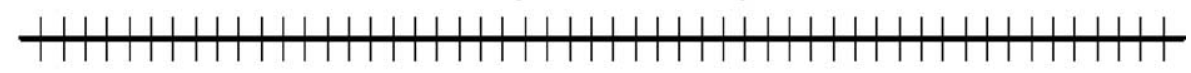

Part 2: Test

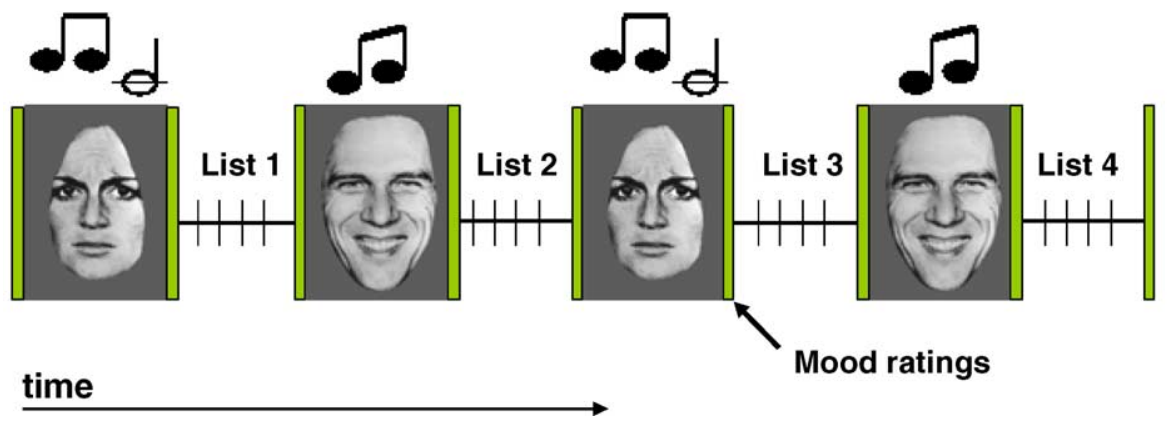

Fig. 2. Order of events in our task: Part 1 was followed immediately by Part 2. Green bars show times of subjective mood ratings. Happy/sad faces show times of corresponding mood induction (the order of happy/sad was reversed for half the subjects). (For interpretation of the references to colour in this figure legend, the reader is referred to the web version of this article.) 
with an SOA of $3 \mathrm{~s}$. The words were broken into four separate lists, each containing 62 of the previously presented words (31 positive and 31 negative) and 28 (14 positive and 14 negative) similarly valenced distracter words which had not been presented at study (i.e., new words). Lists were balanced for mean ANEW valence and arousal scores, and the words within each list were presented in one of two possible pseudo-random orders.

In the $\mathrm{R} / \mathrm{K} / \mathrm{N}$ task (Tulving, 1985), subjects indicated if they remembered a previous experience complete with aspects of the specific context in which it occurred, ( $\mathrm{R}$ response, reflecting recollection), knew that information was familiar without a distinct recollection of prior occurrence ( $\mathrm{K}$ response reflecting familiarity), or identified as a new item not seen at encoding ( $\mathrm{N}$ response). All responses were made by button presses using the right hand. Subjects were asked to respond as accurately as possible while still minimizing response times.

Prior to presentation of each test list, subjects underwent a mood induction procedure consisting of music played for $3.15 \mathrm{~min}$ accompanied by the presentation of a series of standardized emotional faces (Ekman and Friesen, 1976) for $3.5 \mathrm{~s}$ each with a gap of $5 \mathrm{~ms}$ between presentations (Fig. 2, Part 2). The music for 'sad' induction was taken from Albinoni's Adagio in G minor and that for happy was from Coppelia ("Mazurka") by Delibes. The faces were matched in valence to the music, and consisted of the 9 happy and 9 sad expressions presented in a recurring loop. Half of the subjects experienced positive inductions prior to the first and third lists and negative inductions prior to the other lists, and the other half experienced the opposite order. Subjects rated mood levels using a sliding visual analogue scale both immediately after each mood induction and immediately after each test block. The task was presented using a PC running Cogent, a Matlab-based stimulus program. Responses were made using an MR compatible five-button response box.

\section{Autonomic monitoring}

Physiological data were acquired to index states of autonomic arousal that could provide an implicit measure of changes in mood elicited by the mood induction procedure. Throughout the scanning period, we monitored heart rate using pulse oximetry (Nonin 8600 Pulse Oximeter, Nonin Medical, Inc. N. Plymouth, MN, USA). The timing of each pulse and were logged (with slice synchronization signals from the scanner) using CED 1401 data acquisition unit and Spike 3 software (CED, Cambridge Electronic Design Limited, Cambridge, UK). For each test period, we derived measures of mean heart rate and used heart rate variability and power spectral analyses to quantify parasympathetic/sympathetic tone and balance drive (using a Matlab-based software package G.BSanalyze, g.tec-Guger Technologies OEG). The latter provided a more sensitive account of autonomic influences on the heart.

\section{Functional imaging}

Functional data were acquired using a Siemens Allegra 3T scanner. We acquired T2* weighted echo-planar images (EPI) composed of 44 2-mm-thick slices (TR $2.86 \mathrm{~s}$ ), giving whole brain coverage and using a sequence optimized for sensitivity to responses in the orbitofrontal cortex and medial temporal lobe structures (Deichmann et al., 2003). Acquisition was tilted at an oblique angle of $30^{\circ}$ to the anterior-posterior commissure line, and a preparation pulse (duration $1 \mathrm{~ms}$, amplitude of $-2 \mathrm{mT} / \mathrm{m}$ ) was used in the slice selection direction. Data were acquired continuously during 5 different sessions: 300 volumes at encoding, and 141 volumes in each of the four retrieval epochs. High-resolution T1 weighted structural images were also acquired.

\section{Analysis of functional imaging data}

Functional data were analyzed using SPM2 (http://www.fil.ion. ucl.ac.uk/spm/spm2.html). The first 6 volumes of each scanning session were discarded to allow for $\mathrm{T} 1$ saturation effects, and the remaining volumes were realigned and corrected for slice timing differences. A mean image was produced for each session, and was volumetrically normalized to the SPM2 EPI template. The images were then transformed to the MNI canonical brain using these parameters and smoothed with an 8-mm kernel.

\section{Modeling, contrasts, and analyses}

To determine neural responses, the data were examined using a two-level mixed-effects analysis. At the first level, data were modeled separately for each subject. In study (encoding) data, all three possible subsequent response options $(\mathrm{R} / \mathrm{K} / \mathrm{N})$ were modeled using separate regressors for each of the four categories: [positive words positive mood (ePP), positive words negative mood $(\mathrm{ePN})$, negative words negative mood $(\mathrm{eNN})$, and negative words positive mood (eNP)], giving 12 possible combinations. In test (retrieval) data, all six possible response options $(\mathrm{R} / \mathrm{K} / \mathrm{N}$ for old items and $\mathrm{R} / \mathrm{K} / \mathrm{N}$ for new items), were modeled for each of the four categories: [positive words positive mood (PP), positive words negative mood $(\mathrm{PN})$, negative words negative mood $(\mathrm{NN})$, and negative words positive mood (NP)], giving 24 possible combinations. Missed responses were modeled separately in both study and test data. Parameter estimates reflecting the height of the hemodynamic response function for each regressor were calculated at each voxel.

Contrasts of parameter estimates from individual subject analyses were entered into second-level group models. The images resulting from these contrasts were used in four distinct random effects analyses. First (analysis 1), to isolate retrieval-related activity, we tested for brain regions where activity was greater for correctly recollected (RR) compared to correctly rejected (CR) items. To ensure that this retrieval-related activity was independent of mood or word valence, we performed a conjunction analysis (testing for commonalities) across all four categories [PN, PP, NN, $\mathrm{NP}]$ of $[\mathrm{RR}>\mathrm{CR}]$ images. Second (analysis 2), to test the prediction that emotional areas linked to a memory during encoding are reactivated during mood congruence at retrieval, we performed a conjunction searching for commonalities in activity isolated by contrasts at study and at test. At study, our contrast highlighted brain regions where activity predicted subsequent memory in a mood congruent context by isolating the interaction between a subsequent memory effect [eRR $>\mathrm{eF}$, (where eRR includes subsequent RR words and $\mathrm{eF}$ includes words with subsequent $\mathrm{K}$ or $\mathrm{N}$ responses), and the influence of congruent mood at retrieval ([ePP $>\mathrm{ePN}]$ for positive words and [eNN $>\mathrm{eNP}]$ for negative words). At test, our contrast highlighted brain regions where activity reflected mood congruent recollection by comparing RR responses in congruent and incongruent moods ([PP $>$ PN] for positive words and $[\mathrm{NN}>\mathrm{NP}]$ for negative words). Our conjunction was performed across these two contrasts, and was calculated separately for each valence in order to allow identification of 
emotion specific nodes. Third (analysis 3), to determine the influence of mood congruence upon correct and attempted recollection of valenced information, we performed a conjunction between the simple effects of positive $[\mathrm{PP}>\mathrm{PN}]$ and negative $[\mathrm{NN}>$ $\mathrm{NP}$ ] mood, computing this separately for RR and CR responses. Our fourth analysis (analysis 4) determined the relative influence of mood congruence upon $\mathrm{RR}$ and $\mathrm{CR}$ responses using direct comparisons of the results from analysis 3, [RR congruence $>$ $\mathrm{CR}$ congruence] and [CR congruence $>\mathrm{RR}$ congruence].

All statistical images were calculated using SPM $t$ tests. Due to the stringent nature of our second-level random effects analysis, and because of the subtle response expected in higher brain regions influenced by mood manipulations, we used a statistical threshold of $P<0.001$, uncorrected for multiple comparisons, unless otherwise stated. We had no a priori hypothesis about the extent of responding clusters and therefore did not use an extent threshold. Our analysis provides a robust descriptive account of the neural substrates of mood congruency.

\section{Results}

\section{Subjective rating}

Prior to the first mood induction, the mean subjective rating (calculated across the 14 subjects for whom fMRI data was examined) was 5, indicating a slightly positive mood. Further subjective ratings (Fig. 3A) show that our mood manipulation was successful for both positive and negative valence. We performed a 2 $($ mood $) \times 2$ (time of rating) way ANOVA to determine the influence of mood inductions and time of rating upon subjective mood ratings. This revealed a main effect of $\operatorname{mood}(P=0.011)$ indicating a significant difference between subjective ratings occurring after positive and negative inductions, a main effect of time $(P=0.005)$ indicating a significant difference between ratings taken at the start and end of a test block, and no significant interaction $(P=0.415)$.

\section{Heart rate data}

Heart-rate data were analyzed for 15 subjects (heart rate data for the other 3 subjects was lost due to technical difficulties) to test for autonomic correlates of positive and negative mood states induced by the mood induction procedure. Differences in the mean subject heart rate did not reach threshold significance between positive and negative mood conditions. However, there were significant differences in more sensitive indices of sympathetic and parasympathetic influences on cardiac response, i.e., heart rate variability and frequency domains across the two moods. Heart rate variability, here measured from the standard deviation of interpulse intervals, is a surrogate measure of parasympathetic to sympathetic balance. We observed significantly reduced heart rate variability associated with the positive mood (mean of $135 \mathrm{~ms}$ in the positive mood compared to $150 \mathrm{~ms}$ in the negative mood; sine test $P=0.016$ ) across all subjects. Power spectral analyses enables further quantification of autonomic tone, reflected in the different oscillatory frequencies of sympathetic, parasympathetic, and humoral influences on heart rate. Induction of a positive mood was associated with an increase in low- and high-frequency components of heart rate variability $(P=0.016$ and 0.016 , respectively), indicating enhancement of both parasympathetic and sympathetic neural drive to the heart during positive emotional 'excitement'. In contrast, negative mood was associated with relative increase in total frequency power $\left(1265 \mathrm{~ms}^{2}\right.$, compared to $1000 \mathrm{~ms}^{2}$ in the positive mood; sine test $\left.P=0.042\right)$, and particularly very low frequency components $(P=0.01)$ suggestive of a shift in cardiac control to circulating influences. Together, the

\section{A) Subjective mood ratings}

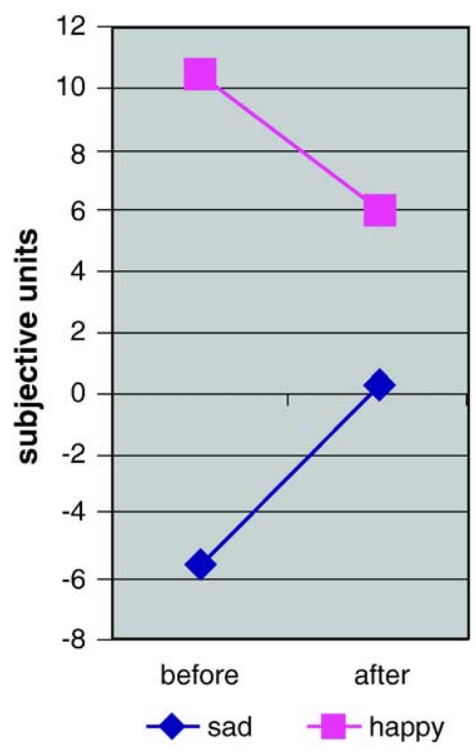

B)

\section{Performance (R Hit - R False Alarm)}

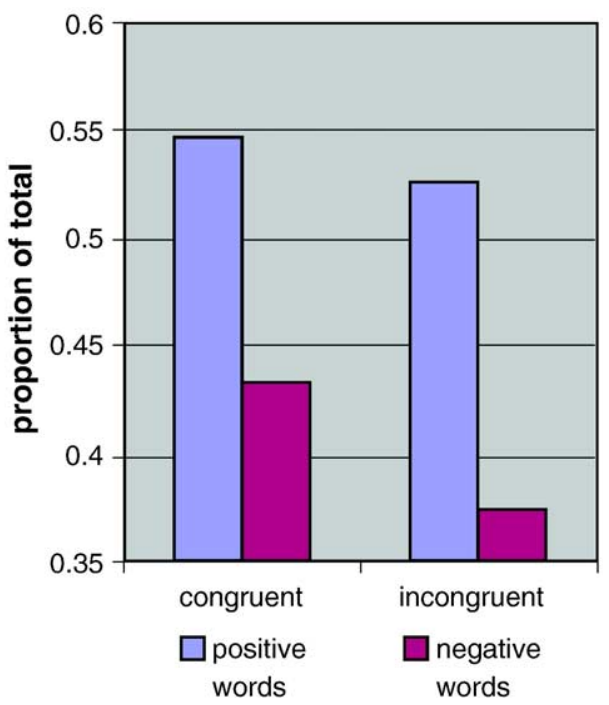

Fig. 3. Panel A shows mean mood ratings in subjective units both immediately before and immediately after the memory test block. Zero indicates a neutral mood, while negative deviations indicate negative and positive deviations indicate positive mood ratings. Panel B shows recollection accuracy for congruent and incongruent trials. 
physiological data provide objective empirical confirmation of the efficacy of the mood induction processes, demonstrating dissociable states of autonomic arousal in positive and negative moods (Task Force of the European Society of Cardiology and the North American Society of Pacing and Electrophysiology, 1996).

\section{Memory performance}

Our data showed a trend towards mood congruent facilitation of recollection, as indexed by $\mathrm{R}$ responses. Recollection accuracy $(\mathrm{R}$ hits $-\mathrm{R}$ false alarms) (Tulving, 1985) scores were calculated for each subject and used in a 2 (word valence) $\times 2$ (mood congruence) way ANOVA. Results showed a significant main effect of word valence, $P=0.01$, with positive words better remembered than negative words, a trend towards a main effect of mood congruence $(P=0.068)$, and a significant interaction $(P=$ 0.036 ) between the two factors (see Fig. 3B). Post hoc two-tailed $t$ tests reveal a significant influence of congruence on memory for negatively valenced words $(P=0.01)$, but not for positively valenced words $(P=0.38)$. Due to high levels of $\mathrm{K}$ false alarms (mean 31\%), recognition accuracy scores on the $\mathrm{K}$ responses did not differ significantly from zero $(P=0.4)$ and thus could not be used to draw conclusions about the influence of congruence upon familiarity. Consistent with earlier work (Brewer et al., 1998; Otten and Rugg, 2001; Wagner et al., 1998), K responses were therefore combined with misses as a measure of forgetting.

\section{Functional imaging results}

\section{Conjunction of all mood and valence conditions}

To isolate the neural activity associated with successful retrieval independent of mood and word valence, we performed a conjunction across results of the $\mathrm{R}>\mathrm{CR}$ contrast in all four word mood combinations (analysis 1). This revealed significant activity
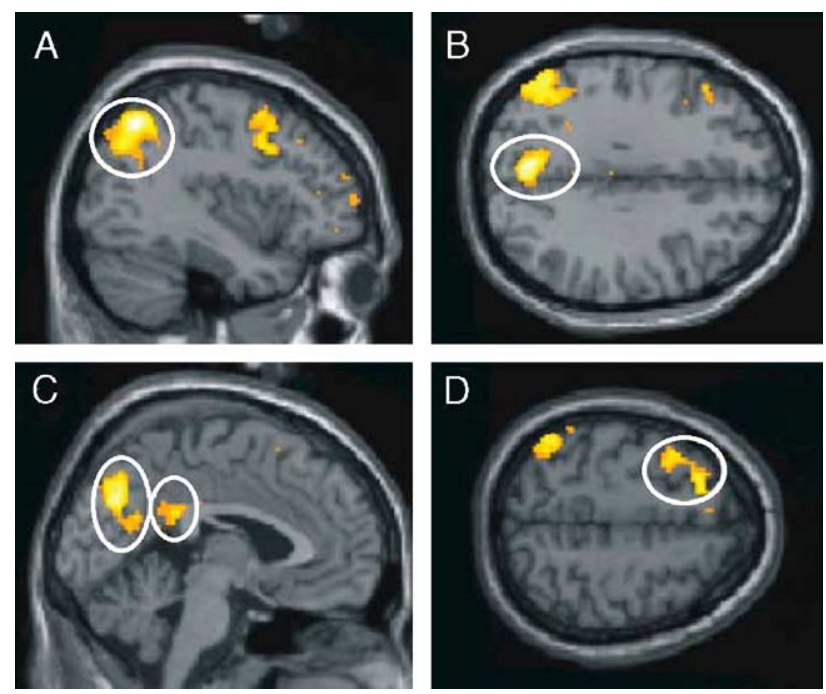

Fig. 4. Results from conjunction across the $[\mathrm{R}>\mathrm{CR}]$ contrasts (analysis 1 ), showing neural activity associated with successful retrieval independent of mood and word valence. Data was thresholded at a whole brain corrected value of $P<0.05$. (A) Inferior parietal cortex, (B) superior parietal cortex, (C) posterior cingulate, with activity spreading into the precuneus, activity in posterior cingulate is also visible (smaller circle), and (D) dorsolateral prefrontal cortex. in left inferior parietal cortex, medial superior parietal cortex, dorsolateral prefrontal cortex, posterior cingulate, and in right cerebellar hemisphere and vermis (see Fig. 4 and Table 1A). These results are broadly in line with previous findings for episodic retrieval (Fletcher and Henson, 2001; Henson et al., 1999).

\section{Valence-specific correlates of predictive encoding and mood congruence at retrieval}

To test the prediction that emotional areas linked to a memory during encoding are reactivated by a mood state during congruent retrieval, we performed a conjunction across study and test data for each valence (analysis 2). Our contrast in study data examined the influence of congruent mood at retrieval upon a subsequent memory effect and was calculated separately for words of each valence. Our contrast at test was a comparison between RR responses in congruent and incongruent moods, again calculated separately for each valence. Areas responding to the conjunction across these two contrasts included two regions known for involvement in emotional processing, the subgenual cingulate for positive words (see Table 1B/Figs. 5A-C), and the right posterior lateral orbitofrontal cortex for negative words (see Table 1C/Figs. 5D-F).

\section{Valence-independent mnemonic correlates of mood congruence}

To determine the neural correlates of congruence in successful and attempted retrieval independent of valence, we calculated the influence of congruence upon positive and negative words separately and then performed a conjunction across the two valences (analysis 3). For RR trials, none of observed activity fell within the episodic memory system (see Table 1D). For CR trials, congruence-related activity occurred in many areas associated with episodic memory, including right dorsolateral prefrontal cortex, precuneus, and posterior cingulate (see Table 1E).

The marked difference between congruence-related responses for RR and CR trials suggests that congruence-related alterations in memory processing occur at the level of attempted recall rather than as an aspect of successful recollection itself. To check the significance of this distinction, we performed a direct comparison between the congruence related activities found in RR and CR trials [RR congruence $>\mathrm{CR}$ congruence], (analysis 4). No brain area showed greater activity for congruence in RR responses; however, a number of regions, including dorsolateral prefrontal cortex, precuneus, anterior insula, and posterior cingulate (see Fig. 6 and Table 1F), were significantly activated by the opposite contrast.

\section{Discussion}

The primary goal of this study was to investigate the neural basis of an associative hypothesis for mood congruent memory facilitation. Specifically, we proposed that emotionally relevant brain regions in which encoding activity predicts subsequent mood congruent retrieval would also reactivate in response to a congruent mood at retrieval. Our results show that two structures known to be involved in emotional processing respond in precisely this manner and these areas therefore provide candidate loci for the valencespecific nodes proposed by our associative model. The secondary goal of this study was to determine how the episodic memory system responds to mood congruence, independent of valence. Our results show increased activity throughout much of this system during attempted but not successful retrieval, thus suggesting that 
Table 1

Coordinates of observed activation

\begin{tabular}{|c|c|c|c|c|}
\hline$Z$ value & $x$ & $y$ & $z$ & Anatomical locus \\
\hline \multicolumn{5}{|c|}{$\begin{array}{l}\text { (A) Conjunction of all four mood/valence combinations for the } \\
[R>C R] \text { contrast (analysis } 1)\end{array}$} \\
\hline$>8$ & -38 & -60 & 50 & inferior parietal \\
\hline$>8$ & -6 & -70 & 32 & superior parietal \\
\hline 6.3 & -12 & 58 & 26 & $\begin{array}{l}\text { superior frontal gyrus } \\
\text { (frontal pole) }\end{array}$ \\
\hline 7 & -18 & 24 & 56 & middle frontal gyrus (DLPFC) \\
\hline 6.2 & -2 & -38 & 26 & posterior cingulate \\
\hline 5.3 & -8 & -48 & 40 & posterior cingulate \\
\hline 6.6 & -62 & -50 & -4 & middle temporal gyrus \\
\hline 5.6 & -64 & -54 & 12 & middle temporal gyrus \\
\hline 6.1 & 10 & -84 & -26 & cerebellar vermis \\
\hline 6 & 32 & -66 & -32 & cerebellar hemisphere \\
\hline 5.9 & -48 & 48 & -4 & cerebellar hemisphere \\
\hline
\end{tabular}

(B) Conjunction of encoding and retrieval data, POSITIVE words (analysis 2)

$\begin{array}{lrrrl}3.3 & 2 & -52 & -10 & \text { cerebellum } \\ 3.3 & -46 & 50 & 2 & \text { middle frontal gyrus (DLPFC) } \\ 3.3 & 22 & 32 & 46 & \text { superior frontal gyrus (DLPFC) } \\ 3.2 & 30 & -2 & 54 & \text { middle frontal gyrus (DLPFC) } \\ 3.2 & -22 & -66 & -2 & \text { cuneus/precuneus } \\ 3.2 & 8 & 40 & -4 & \text { subgenual cingulate } \\ 3.1 & 34 & 8 & 54 & \text { middle frontal gyrus (DLPFC) }\end{array}$

(C) Conjunction of encoding and retrieval data, NEGATIVE words (analysis 2)

\begin{tabular}{lrrrl}
4.1 & 26 & -90 & -22 & cerebellum \\
4 & 0 & 38 & 44 & medial superior frontal gyrus \\
3.7 & -46 & -26 & -14 & middle temporal gyrus \\
3.7 & 32 & -60 & -20 & lateral cerebellum \\
3.7 & -34 & -70 & -22 & lateral cerebellum \\
3.7 & 34 & -66 & 60 & inferior parietal \\
3.6 & -14 & -76 & 58 & superior parietal \\
3.6 & -52 & -46 & 56 & inferior parietal \\
3.6 & 36 & 30 & -14 & lateral orbitofrontal \\
3.6 & 38 & 30 & 42 & middle frontal gyrus (DLPFC) \\
3.5 & -38 & 40 & 4 & inferior frontal gyrus (VLPFC) \\
3.5 & 26 & -76 & -20 & lateral cerebellum \\
3.4 & 22 & -74 & -26 & lateral cerebellum \\
3.4 & 14 & -72 & 58 & superior parietal \\
3.4 & -2 & 40 & 54 & medial superior frontal gyrus \\
3.3 & -64 & -42 & 28 & inferior parietal \\
3.2 & -44 & 18 & -10 & anterior insula \\
3.2 & 22 & 24 & -8 & anterior insula \\
3.2 & 18 & 66 & 0 & frontal pole \\
3.2 & 10 & -68 & 38 & superior parietal \\
3.2 & 42 & -32 & 2 & superior temporal gyrus \\
3.1 & -34 & 6 & -26 & middle temporal gyrus \\
3.1 & 6 & -14 & 30 & corpus callosum \\
3.1 & 4 & 24 & 44 & medial superior frontal gyrus \\
& & & & \\
$(D)$ & Congruent & $R R$ & incongruent $R R$ & (analysis 3) \\
3.8 & 40 & -48 & -38 & lateral cerebellum \\
3.7 & 6 & -14 & -12 & brainstem \\
3.5 & -88 & -22 & lateral cerebellum \\
3.4 & 32 & -12 & lateral orbitofrontal \\
3.4 & 86 & 28 & inferior frontal gyrus (VLPFC) \\
3.3 & -10 & lateral orbitofrontal \\
3.2 & & -16 & ventral striatum \\
\hline & & &
\end{tabular}

Table 1 (continued)

\begin{tabular}{lrrrl}
\hline$Z$ value & $x$ & $y$ & \multicolumn{2}{l}{ Anatomical locus } \\
\hline (E) & Congruent $C R$ & incongruent $C R$ & (analysis 3) \\
3.8 & 30 & 42 & -4 & lateral orbitofrontal \\
3.7 & 20 & -52 & 18 & precuneus \\
3.7 & -42 & -66 & -26 & cerebellum \\
3.5 & 28 & 46 & 36 & middle frontal gyrus (DLPFC) \\
3.4 & 44 & 8 & 30 & middle frontal gyrus \\
3.4 & 30 & 24 & 4 & insula \\
3.4 & -8 & -38 & 34 & posterior cingulate \\
3.3 & 42 & 20 & 2 & insula \\
3.2 & 56 & 26 & 20 & inferior frontal gyrus (VLPFC) \\
3.2 & 10 & 46 & 48 & medial superior frontal gyrus \\
3.2 & 56 & -46 & 42 & inferior parietal lobule \\
3.2 & -38 & -56 & -30 & cerebellar hemisphere \\
3.2 & 32 & 10 & 48 & middle frontal gyrus (DLPFC) \\
3.1 & 56 & 30 & 8 & inferior frontal gyrus (VLPFC) \\
3.1 & -22 & -10 & -8 & amygdalar tail
\end{tabular}

(F) [congruent $C R>$ incongruent $C R]>$ [congruent $R>$ incongruent $R$ ] exclusively masked by [congruent $C R>$ incongruent $C R$ ] (analysis 4)

\begin{tabular}{rrrrl}
3.7 & 32 & 44 & 22 & middle frontal gyrus (DLPFC) \\
3.6 & -8 & -40 & 30 & posterior cingulate \\
3.5 & 42 & 20 & 4 & anterior insula \\
3.5 & 18 & -52 & 18 & precuneus \\
3.5 & -38 & 50 & 16 & middle frontal gyrus (DLPFC) \\
3.4 & 22 & -20 & 0 & thalamus (vpi) \\
3.3 & 28 & 26 & 4 & anterior insula \\
3.2 & -20 & -8 & -8 & brainstem \\
3.2 & -12 & 10 & 16 & head of caudate \\
3.2 & 54 & 30 & 8 & inferior frontal gyrus (VLPFC) \\
3.2 & 28 & 44 & -2 & orbitofrontal cortex \\
3.2 & -18 & 52 & -4 & orbitofrontal cortex \\
3.2 & 6 & -22 & 4 & thalamus/gp \\
3.2 & -24 & 36 & 26 & middle frontal gyrus (DLPFC) \\
3.1 & 12 & 60 & 22 & medial superior frontal gyrus \\
3.1 & 16 & -44 & 32 & posterior cingulate \\
3.1 & -4 & 8 & 56 & medial superior frontal gyrus \\
\hline
\end{tabular}

This table shows $Z$ values and $x / y / z$ coordinates $(\mathrm{mm})$ for peaks of activity. (A) All peaks above a statistical threshold of $P<0.05$ corrected are shown. (B-E) All peaks above a statistical thresholded at $P<0.001$ uncorrected are shown. DLPFC $=$ dorsolateral prefrontal cortex, VLPFC $=$ ventrolateral prefrontal cortex, gp = globus pallidus.

congruence influences memory processes at the level of search elicited by new items rather than at the level of successful episodic recollection.

\section{Neural correlates of valence-dependent mood congruence}

The regions implicated as loci for valence-specific associative memory nodes were subgenual cingulate for positive valence and posterior-lateral orbitofrontal cortex for negative valence. The involvement of these regions in emotional processing is well established. Subgenual cingulate has been shown to respond to both positive and negative stimuli (see Phan et al., 2002; Phillips et al., 2003 for reviews), and is known to be involved in reward representation (Elliott et al., 2002), the expression of pathological mood (Mayberg et al., 1999), and in mood disorders (Drevets et al., 1997). Lateral orbitofrontal cortex has repeatedly been associated with punishment (Elliott et al., 2004; O'Doherty et al., 2001, 2003). Because these findings were revealed by a conjunction across two contrasts, however, it is important to 

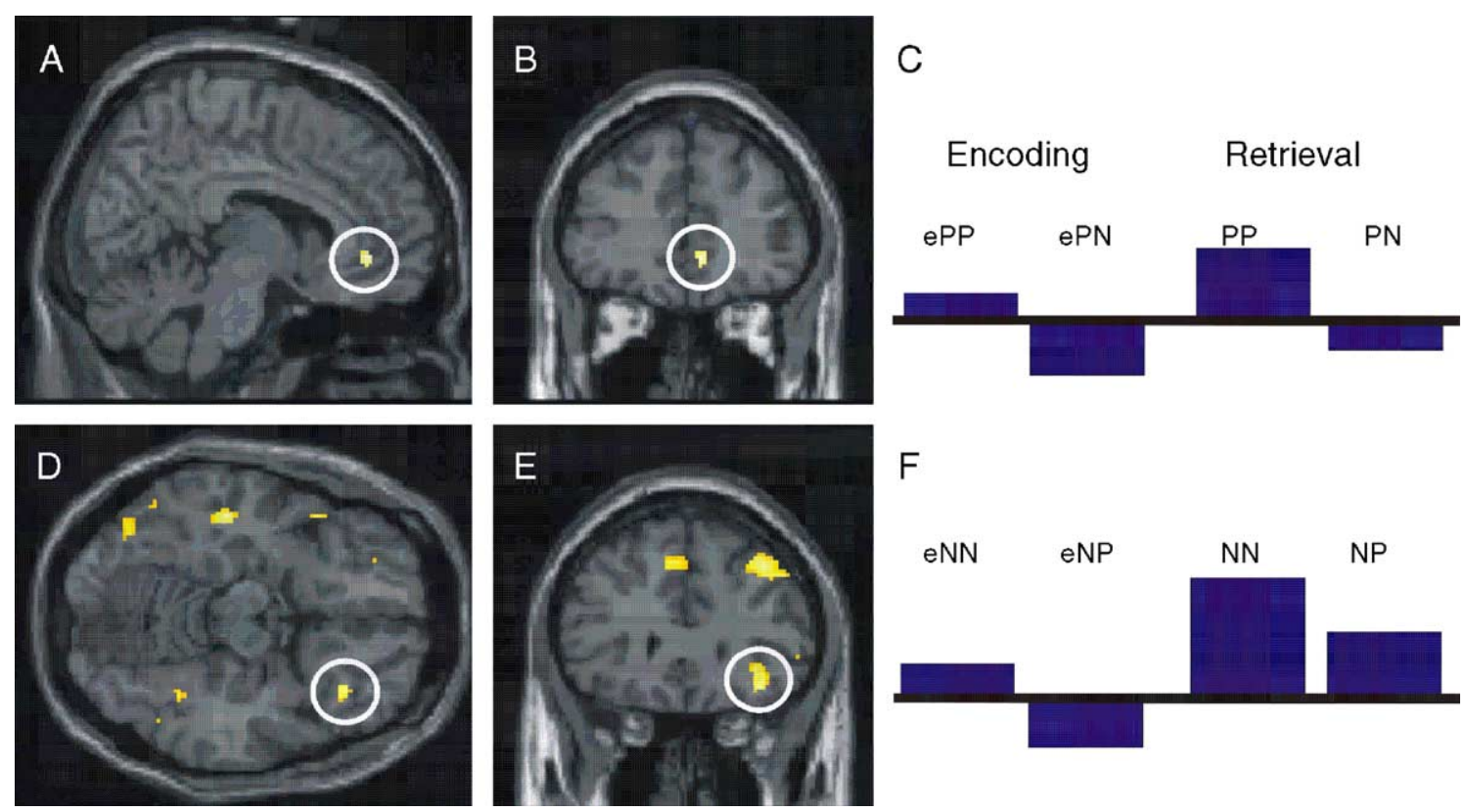

Fig. 5. Results from conjunctions across encoding and retrieval data (analysis 2), showing that emotional areas linked to a memory during encoding are reactivated during mood congruence at retrieval. Data were thresholded at $P<0.005$ uncorrected. Panels A and B show activation in the right subgenual cingulate in associated with positive words, while panel $\mathrm{C}$ shows the associated parameter in ePP and ePN conditions at encoding and in PP and PN conditions at retrieval. Panels D and E show activity in the left posteriolateral orbitofrontal cortex while panel F shows the associated parameter estimates in eNN and eNP conditions at encoding and NN and NP conditions at retrieval.

discuss both components in detail in order to interpret the results.

The contrast used at encoding was an interaction between subsequent recollection and the mood in which this recollection occurred. This comparison aimed to isolate activity associated with aspects of encoding which led to a bias for facilitated recollection in a congruent mood. It thus relied upon the assumption that the
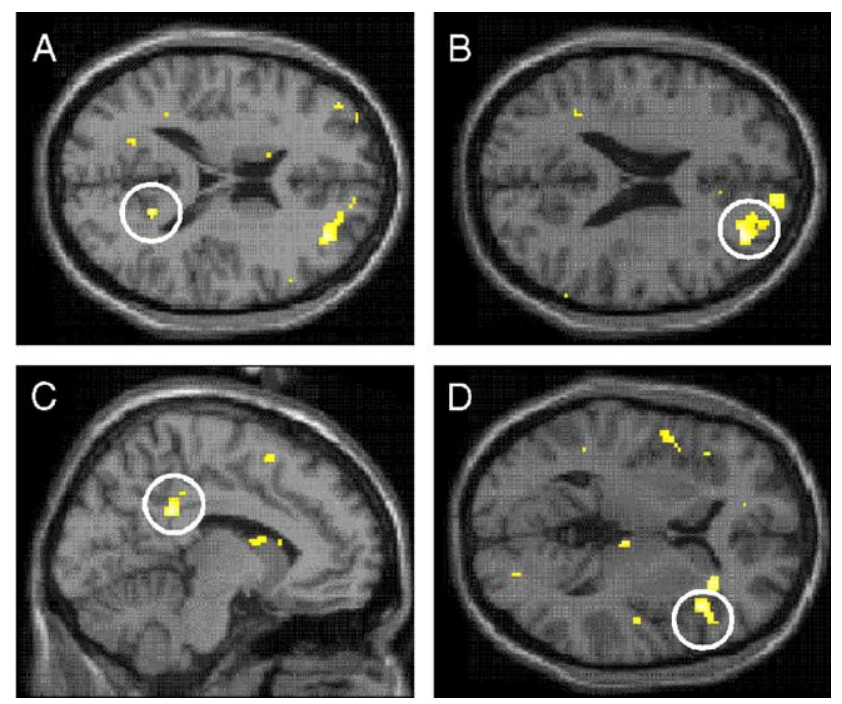

Fig. 6. Results from comparison of congruency related activity in RR and $\mathrm{CR}$ responses [CR $>\mathrm{RR}$ ] (analysis 4), showing areas which respond more strongly to congruency in $\mathrm{CR}$ than RR responses. Data was thresholded at $P>0.005$ uncorrected. Areas shown are: (A) right precuneus, (B) right dorsolateral prefrontal cortex, (C) left posterior cingulate, (D) right anterior insula. way a word is encoded can influence the way it will be recalled (Mitchell et al., 2004; Schott et al., 2002) and further, upon the idea that differential encoding can predispose material to facilitated recollection in congruent as compared to incongruent moods. A bias of this type could be due to any factor influencing encoding, for instance, mood, arousal state, or characteristics of individual words. It is important to note, however, that this contrast would be confounded if any of the resulting activity were due to effects reflecting the stronger encoding necessary for subsequent memory in incongruent conditions. Previous work has shown that greater activity in the episodic memory system can be associated with subsequent forgetting (Otten and Rugg, 2001; Wagner and Daachi, 2001) and it follows that more effective encoding may be associated with deactivations in that system. Because there is no reason to expect that this type of processing should occur in a valence-specific manner, we were able to check this possibility by looking for valence-independent activity in the subgenual cingulate and posterior-lateral orbitofrontal cortex using a conjunction across the subsequent memory effects $[\mathrm{eRR}>\mathrm{eF}]$ in positive and negative words. The results showed no activity in either structure at a threshold of $P<0.001$ uncorrected, arguing against the possibility that the reported activity could be explained by deactivations of this type.

The retrieval portion of our conjunction examined the simple effects of congruence upon RR responses. Thus, areas highlighted in this contrast were more active during congruent than incongruent retrieval of words with a particular valence. This comparison aimed to isolate regions responding to positive or negative mood during recollection in congruent circumstances. As with the encoding data, we checked for the possibility that observed activities were due to deactivations associated with incongruent retrieval by performing a conjunction across the RR congruence responses from both valences. This check revealed no activity in 
either of the emotionally relevant target regions at $P<0.001$ uncorrected and thus argued against the idea that the reported activity could be explained by encoding-related deactivations.

Subgenule cingulate and posteriolateral orbitofrontal cortex demonstrated shared activity across both encoding and retrieval contrasts. Thus, neural responses in these regions were associated both with encoding activity predicting subsequent recollection in a congruent mood and with retrieval activity indexing correct congruent recollection. This shared pattern of activity is in good keeping with the predictions of our associative memory hypothesis (Bower, 1981), see Fig. 1, for the behavior of valence-specific nodes. Because subsequent mood congruent facilitation can only occur if these nodes are active at encoding, our model proposes that activity in these areas during encoding will be predictive of subsequent memory in congruent moods. Because mood congruent facilitation can only occur when material is remembered in a congruent mood, our model further predicts greater activity in emotional nodes during congruent than incongruent recall. Since subgenual cingulate and lateral orbitofrontal activity follows precisely this pattern, these regions are prime candidates for the facilitatory role postulated by our model (Lewis and Critchley, 2003). Further studies will be required to determine whether or not these activities are in fact associated with facilitation. The close functional anatomical connectivity of caudal orbitofrontal cortex and subgenual cingulate cortex with autonomic centers in hypothalamus and brainstem (Ongur et al., 1998), and our confirmation that positive and negative moods produce differential states of autonomic arousal, suggests an additional psychophysiological component to mood congruent memory. This is the possibility that peripheral somatic arousal states may provide a referential context for retrieval.

Because the medial prefrontal cortex is known to be involved in self-referential processing (Fossati et al., 2003, 2004; Kelley et al., 2002), it is important to consider the possibility that the observed medial prefrontal activity was due to self-referential processing rather than positive congruence. To this end, we modeled activity associated with self-referential responding. This analysis showed no activity in medial prefrontal cortex. Furthermore, when word valence was modeled parametrically from most positive words to most negative words, we found strong activity in the anterior cingulate even when selfreferential responding was modeled out. In lieu of these results, we are confident that the medial prefrontal activity reported in this study is associated with the processing of positive stimuli and not with self-referential processing.

\section{Valence-independent correlates of mood congruence}

A secondary goal of this study was to describe how the episodic memory system responds to mood congruence regardless of valence. Because memory performance is facilitated in mood congruent conditions, it is reasonable to infer that those parts of the system in which activity alters during congruence are associated with the observed improvement. We were able to distinguish between congruence-linked activity occurring during processes supporting retrieval attempts and during processes dependent upon retrieval success by examining correctly rejected (CR) and correctly recollected (RR) trials separately. Although RR responses involve retrieval attempts as well as post-retrieval processing, comparing the facilitated retrieval processes in congruent $\mathrm{RR}$ responses to retrieval processes which were successful despite occurring in the incongruent condition cannot isolate the neural correlates of facilitation leading to success. CR responses, on the other hand, include both those attempts which would have resulted in correct retrieval had the cue been seen at encoding, and those which would not have succeeded in retrieving information. Because the cues presented on CR trials are new, the responses associated with attempted retrieval are not confounded by processes associated with recognition. Our observation that parts of the episodic memory system were activated by congruence in CR but not in RR responses, and that this difference in activity was significant when $\mathrm{CR}$ congruence and RR congruence responses were compared, therefore suggests that mood congruence acts upon the processes supporting retrieval attempts rather than those dependent upon successful retrieval.

Because two of the regions which responded to congruence in CR trials are believed to have quite specific roles in memory, we can draw upon existing knowledge to speculate about the processes influenced in mood congruent facilitation. The precuneus has repeatedly been associated with mental imagery during memory tasks (Fletcher et al., 1995, 1996; Ghaem et al., 1997). Our observation of congruence-related activity here therefore suggests an involvement of such imagery in the facilitation effect. The right dorsolateral prefrontal cortex is thought to participate in postretrieval monitoring of information in working memory (Rugg et al., 2003; Sharp et al., 2004). The greater activity in this structure for congruent as compared to incongruent CR responses therefore suggests increased monitoring during congruent retrieval attempts. Under a signal detection framework, it has been suggested that more monitoring occurs when information is nearer the threshold for retrieval and subjects are less certain about their responses (Henson et al., 2000). If the criterion for classifying items as old is lower in congruent moods, more new items will be closer to the response threshold and more monitoring may therefore be required. Because subjects more readily classify stimuli as old when the criterion is shifted downwards, this change in strategy should be accompanied by an increase in both hits and false alarms. Combined across $\mathrm{R}$ and $\mathrm{K}$ responses, our data show a strong trend towards this pattern in congruent conditions, with a onetailed $t$-test probability of $P=0.09$ for correct responses and of $P=$ 0.07 for false alarms for the comparison of congruence to incongruence. Taken together, these findings suggest that enhanced capacity for mental imagery and enhanced monitoring of retrieval attempts, perhaps as a result of a rightward shift in response criterion, may be two of the processes underlying mood congruent memory facilitation.

\section{Relating the associative memory hypothesis to generalized influences of congruence}

Our results demonstrate both that brain regions known for emotional processing respond in a manner reminiscent of the emotion-specific nodes posited by the associative memory framework, and that a widespread up-regulation of activity occurs in regions known to be involved in the episodic memory system during attempts to retrieve information in mood congruent conditions. Although these can be thought of as discreet findings, it is tempting to propose a causal link between them. One possibility is that activity in the emotion-specific nodes somehow leads to the observed up-regulation of processes involved in attempted retrieval. This is an adaptation of the associative memory hypothesis, as that framework proposes a more direct interaction 
between emotional nodes and memory. Further experimentation is required to examine the plausibility of such conjecture.

\section{Acknowledgments}

This work was carried out under the EU FET Program, PRESENCIA IST-2001-37927. H.D.C. and R.J.D. are supported by the Wellcome Trust. We are grateful to Leun Otten for helpful comments on the manuscript, to the technical staff and analysis group at the FIL for invaluable assistance, and to Christoph Guger for processing the autonomic data.

\section{References}

Baker, S.C., Frith, C.D., Dolan, R.J., 1997. The interaction between mood and cognitive function studied with PET. Psychol. Med. 27, $565-578$.

Blaney, P.H., 1986. Affect and memory: a review. Psychol. Bull. 99, $229-246$

Bower, G.H., 1981. Mood and memory. Am. Psychol. 36, 129-148.

Bradley, M.M., Lang, P.J., 1999. Affective norms for English words (ANEW): stimuli, instruction manual and affective ratings. C-1. The Center for Research in Psychophysiology, University of Florida, Gainesville, FL. Technical report. Ref Type: Report.

Brewer, J.B., Zhao, Z., Desmond, J.E., Glover, G.H., Gabrieli, J.D.E., 1998. Making memories: brain activity that predicts how well visual experience will be remembered. Science 281, 1185-1187.

Deichmann, R., Gottfried, J.A., Hutton, C., Turner, R., 2003. Optimized EPI for fMRI studies of the orbitofrontal cortex. NeuroImage 19, $430-441$.

Drevets, W.C., Pice, J.L., Simpson, J.R., Todd, R.D., Reic, T., Vannier, M., Raichle, M.E., 1997. Subgenual prefronttal cortex abnormalities in mood disorders. Nature 386, 824-827.

Ekman, P., Friesen, W.V., 1976. Pictures of Facial Affect. Consulting Psychologists' Press, Palo Alto, CA.

Elliott, R., Rubinsztein, J.S., Sahakian, B.J., Dolan, R.J., 2002. The neural basis of mood-congruent processing biases in depression. Arch. Gen. Psychiatry 59, 597-604.

Elliott, R., Newman, J.L., Longe, O.A., William Deakin, J.F., 2004. Instrumental responding for rewards is associated with enhanced neuronal response in subcortical reward systems. NeuroImage 21, 984-990

Fletcher, P.C., Henson, R.N., 2001. Frontal lobes and human memory: insights from functional neuroimaging. Brain 124, 849-881.

Fletcher, P.C., Frith, C.D., Baker, S.C., Shallice, T., Frackowiak, R.S., Dolan, R.J., 1995. The mind's eye-precuneus activation in memoryrelated imagery. NeuroImage 2, 195-200.

Fletcher, P.C., Shallice, T., Frith, C.D., Frackowiak, R.S., Dolan, R.J., 1996. Brain activity during memory retrieval. The influence of imagery and semantic cueing. Brain 119 (Pt. 5), 1587-1596.

Fossati, P., Hevenor, S.J., Graham, S.J., Grady, C., Keightley, M.L., Craik, F., Mayberg, H., 2003. In search of the emotional self: an FMRI study using positive and negative emotional words. Am. J. Psychiatry 160, $1938-1945$.

Fossati, P., Hevenor, S.J., Lepage, M., Graham, S.J., Grady, C., Keightley, M.L., Craik, F., Mayberg, H., 2004. Distributed self in episodic memory: neural correlates of successful retrieval of selfencoded positive and negative personality traits. NeuroImage 22, $1596-1604$.

Ghaem, O., Mellet, E., Crivello, F., Tzourio, N., Mazoyer, B., Berthoz, A., Denis, M., 1997. Mental navigation along memorized routes activates the hippocampus, precuneus, and insula. NeuroReport 8 , $739-744$.
Gotlib, I.H., Cane, D.B., 1987. Construct accessibility and clinical depression: a longitudinal investigation. J. Abnorm. Psychol. 96, 199-204.

Gray, J.R., Braver, T.S., Raichle, M.E., 2002. Integration of emotion and cognition in the lateral prefrontal cortex. Proc. Natl. Acad. Sci. U. S. A. 99, 4115-4120.

Henson, R.N., Rugg, M.D., Shallice, T., Josephs, O., Dolan, R.J., 1999. Recollection and familiarity in recognition memory: an event-related functional magnetic resonance imaging study. J. Neurosci. 19, $3962-3972$.

Henson, R.N., Rugg, M.D., Shallice, T., Dolan, R.J., 2000. Confidence in recognition memory for words: dissociating right prefrontal roles in episodic retrieval. J. Cogn. Neurosci. 12, 913-923.

Hornberger, M., Morcom, A.M., Rugg, M.D., 2004. Neural correlates of retrieval orientation: effects of studytest similarity. J. Cogn. Neurosci. 16 (7), 1196-1210.

Kelley, W.M., Macrae, C.N., Wyland, C.L., Caglar, S., Inati, S., Heatherton, T.F., 2002. Finding the self? An event-related fMRI study. J. Cogn. Neurosci. 14, 785-794.

Lewis, P.A., Critchley, H.D., 2003. Mood dependent memory. Trends Cogn. Sci. 7, 431-433.

Maratos, E.J., Dolan, R.J., Morris, J.S., Henson, R.N., Rugg, M.D., 2001. Neural activity associated with episodic memory for emotional context. Neuropsychologia 39, 910-920.

Mayberg, H.S., Liotti, M., Brannan, S.K., McGinnis, S., Mahurin, R.K., Jerabek, P.A., Silva, J.A., Tekell, J.L., Martin, C.C., Lancaster, J.L., Fox, P.T., 1999. Reciprocal limbic-cortical function and negative mood: converging PET findings in depression and normal sadness. Am. J. Psychiatry 156, 675-682.

Mitchell, J.P., Macrae, C.N., Mahzarin, R.B., 2004. Encoding-specific effects of social cognition on the neural correlates of subsequent memory. J. Neurosci. 24, 4912-4917.

Murphy, F.C., Sahakian, B.J., Rubinsztein, J.S., Michael, A., Rogers, R.D., Robbins, T.W., Paykel, E.S., 1999. Emotional bias and inhibitory control processes in mania and depression. Psychol. Med. 29, 1307-1321.

O’Doherty, J., Kringelbach, M.L., Rolls, E.T., Hornak, J., Andrews, C., 2001. Abstract reward and punishment representations in the human orbitofrontal cortex. Nat. Neurosci. 4, 95-102.

O'Doherty, J., Winston, J., Critchley, H., Perrett, D., Burt, D.M., Dolan, R.J., 2003. Beauty in a smile: the role of medial orbitofrontal cortex in facial attractiveness. Neuropsychologia 41, 147-155.

Ongur, D., An, X., Price, J.L., 1998. Prefrontal cortical projections to the hypothalamus in macaque monkeys. J. Comp. Neurol. 30, $480-505$.

Otten, L.J., Rugg, M.D., 2001. When more means less: neural activity related to unsuccessful memory encoding. Curr. Biol. 11, 1528-1530.

Phan, K.L., Wager, T., Taylor, S.F., Liberzon, I., 2002. Functional neuroanatomy of emotion: a meta-analysis of emotion activation studies in PET and fMRI. NeuroImage 16, 331-348.

Phillips, M.L., Drevets, W.C., Rauch, S.L., Lane, R., 2003. Neurobiology of emotion perception I: the neural basis of normal emotion perception. Biol. Psychiatry 54, 504-514.

Robb, W.G., Rugg, M.D., 2002. Electrophysiological dissociation of retrieval orientation and retrieval effort. Psychon. Bull. Rev. 9, $583-589$.

Rugg, M.D., Henson, R.N., Robb, W.G., 2003. Neural correlates of retrieval processing in the prefrontal cortex during recognition and exclusion tasks. Neuropsychologia 41, 40-52.

Schott, B., Richardson-Klavehn, A., Heinze, H.J., Duzel, E., 2002. J. Cogn. Neurosci. 14, 578-592.

Segal, Z.V., Gemar, M., Truchon, C., Guirguis, M., Horowitz, L.M., 1995. A priming methodology for studying self-representation in major depressive disorder. J. Abnorm. Psychol. 104, 205-213.

Sharp, D.J., Scott, S.K., Wise, R.J., 2004. Monitoring and the controlled processing of meaning: distinct prefrontal systems. Cereb. Cortex 14, $1-10$. 
Smith, A.P., Henson, R.N., Dolan, R.J., Rugg, M.D., 2004. fMRI correlates of the episodic retrieval of emotional contexts. NeuroImage $22,868-878$.

Task Force of the European Society of Cardiology and the North American Society of Pacing and Electrophysiology, 1996. Heart rate variability: standards of measurement, physiological interpretation, and clinical use. Circulation 93, 1043-1065.
Tulving, E., 1985. Memory and consciousness. Can. Psychol. 26, 1-12. Wagner, A.D., Daachi, L., 2001. Cognitive neuroscience: forgetting of things past. Curr. Biol. 11, R964-R967.

Wagner, A.D., Schater, D.L., Rotte, M., Koustaal, W., Maril, A., Dale, A.M., Rosen, B.R., Buckner, R.L., 1998. Building memories: remembering and forgetting of verbal experiences as predicted by brain activity. Science $281,1188-1191$. 